\title{
A note on $\Xi_{c}(3055)^{+}$and $\Xi_{c}(3123)^{+}$
}

\author{
Xiang Liu, ${ }^{1, *}$ Chong Chen, ${ }^{1}$ Wei-Zhen Deng, ${ }^{1}$ and Xiao-Lin Chen ${ }^{1, \oplus}$ \\ ${ }^{1}$ Department of Physics, Peking University, Beijing 100871, China
}

(Dated: October 28, 2018)

\begin{abstract}
Babar Collaboration announced two new excited charmed baryons $\Xi_{c}(3055)^{+}$and $\Xi_{c}(3123)^{+}$. We study their strong decays assuming they are D-wave states. Some assignments are excluded by comparing our numerical results with the experimental values of the total widths of $\Xi_{c}(3055)^{+}$ and $\Xi_{c}(3123)^{+}$. We also suggest some possible decay modes, which will be helpful to determine the properties of $\Xi_{c}(3055)^{+}$and $\Xi_{c}(3123)^{+}$.

PACS numbers: 13.30.Eg, 12.39.Jh
\end{abstract}

At the recent 2007 Euro-physics Conference on High Energy Physics, Babar Collaboration reported the preliminary results about the observations of two new excited charmed baryons $\Xi_{c}(3055)^{+}$and $\Xi_{c}(3123)^{+}$in the mass distribution of $\Lambda_{c}^{+} K^{-} \pi^{+}$[1]. Besides these new observations, Babar also confirmed the observation of $\Xi_{c}(2980)^{+}$and $\Xi_{c}(3077)^{+}[2,3]$. The masses and widths of $\Xi_{c}(3055)^{+}$and $\Xi_{c}(3123)^{+}$are

$$
\begin{aligned}
& m_{\Xi_{c}(3055)^{+}}=3054.2 \pm 1.2 \pm 0.5 \mathrm{MeV} / \mathrm{c}^{2}, \\
& \Gamma_{\Xi_{c}(3055)^{+}}=17 \pm 6 \pm 11 \mathrm{MeV} / \mathrm{c}^{2}, \\
& m_{\Xi_{c}(3123)^{+}}=3122.9 \pm 1.3 \pm 0.3 \mathrm{MeV} / \mathrm{c}^{2}, \\
& \Gamma_{\Xi_{c}(3123)^{+}}=4.4 \pm 3.4 \pm 1.7 \mathrm{MeV} / \mathrm{c}^{2} .
\end{aligned}
$$

In order to understand the recently observed $\Lambda_{c}(2880,2940)^{+}, \Xi_{c}(2980,3077)^{+, 0}$, and $\Omega_{c}(2768)^{0}$ [2, 3, 4, 5, 6], we studied the strong decays of the S-wave, Pwave, D-wave, and radially excited charmed baryons using the ${ }^{3} P_{0}$ model systemically 7$]$. (For more details of the ${ }^{3} P_{0}$ model, see Ref. [8, 9, 10, 11, 12, 13, 14, 15, 16, 17, 18, 19]).

In this short note, we analyze the strong decays of $\Xi_{c}(3055)^{+}$and $\Xi_{c}(3123)^{+}$using the same formalism as in Ref. [7], which will be helpful to determine the quantum number of $\Xi_{c}(3055)^{+}$and $\Xi_{c}(3123)^{+}$. Because the parity of these states is even, they are either the first radial excitation or D-wave charmed baryons. In our previous work [7], we studied the total decay width of $\Xi_{c}(3077)^{+}$assuming it's a candidate of the first radial excitation. Because their masses are close, the decay pattern of $\Xi_{c}(3055,3123)^{+}$should be similar to that presented in Ref. 7] if either of them is the radial excitation. In this work, we will not discuss the assignment for $\Xi_{c}(3055,3123)^{+}$(Interested reader can consult Ref. [7]). In the following, we estimate their strong decays if $\Xi_{c}(3055)^{+}$and $\Xi_{c}(3123)^{+}$are candidates of D-wave states. We list the spectrum of D-wave excited spectrum in Fig. 1. We omit the detailed expressions of the strong decays of D-wave charmed baryons derived by this model. Interested readers may consult our former paper [7] for details.

The decay widths of charmed baryons from the ${ }^{3} P_{0}$ model involve several parameters: the strength of quark pair creation from vacuum $\gamma$, the $\mathrm{R}$ value in the harmonic oscillator wave function of meson and the $\alpha_{\rho, \lambda}$ in the baryon wave functions. We follow the convention of Ref. 20] and take $\gamma=13.4$, which is considered as a universal parameter in the ${ }^{3} P_{0}$ model. The $\mathrm{R}$ value of $\pi$ and $K$ mesons is $2.1 \mathrm{GeV}^{-1}$ [20] while it's $R=2.3 \mathrm{GeV}^{-1}$ for the $D$ meson [21]. $\alpha_{\rho}=\alpha_{\lambda}=0.5 \mathrm{GeV}$ for the proton and $\Lambda$ [19]. For S-wave charmed baryons, the parameters $\alpha_{\rho}$ and $\alpha_{\lambda}$ in the harmonic oscillator wave functions can be fixed to reproduce the mass splitting through the contact term in the potential model [22]. Their values are $\alpha_{\rho}=$ $0.6 \mathrm{GeV}$ and $\alpha_{\lambda}=0.6 \mathrm{GeV}$. For $\mathrm{P}$-wave and $\mathrm{D}$-wave charmed baryons, $\alpha_{\rho}$ and $\alpha_{\lambda}$ are expected to lie in the range $0.5 \sim 0.7 \mathrm{GeV}$. In the following, our numerical results are obtained with the typical values $\alpha_{\rho}=\alpha_{\lambda}=$ $0.6 \mathrm{GeV}$. In the following, we listed the numerical results of the strong decays of $\Xi_{c}(3055)^{+}$and $\Xi_{c}(3123)^{+}$in Table IIII.

At present only total widths of $\Xi_{c}(3055,3123)^{+}$are measured experimentally. Through comparing our numerical results with experimental values, we exclude some D-wave assignments for $\Xi_{c}(3055,3123)^{+}$, which are marked by " $\times$ " in Table IIII. In order to fully determine the quantum numbers of $\Xi_{c}(3055,3123)^{+}$, we suggest:

- Search for other possible decay modes of $\Xi_{c}(3055,3123)^{+}$. From Table [III] one notes that some decay modes are forbidden for $\Xi_{c}(3055,3123)^{+}$with several assignments of their quantum numbers, which provides some useful hint for exclusion or confirmation of certain $J^{P}$.

- Measure the ratio between different decay modes $\Xi_{c}^{0} \pi^{+}: \Xi_{c}^{\prime}(0) \pi^{+}: \Xi_{c}^{* 0} \pi^{+}: \Sigma_{c}^{++} K^{-}: \Sigma_{c}^{*++} K^{-}: \Lambda_{c}^{+} K^{0}:$ $D^{+} \Lambda$. Our numerical results show this ratio is different for the different assignment.

\footnotetext{
*Electronic address: xiangliu@pku.edu.cn

${ }^{\dagger}$ Electronic address: xlchen@th.phy.pku.edu.cn
} 
(a) $l_{\rho}=0, l_{\lambda}=2$

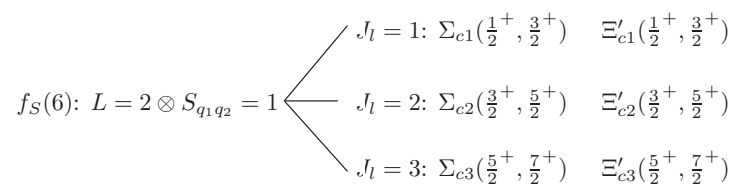

$f_{A}(\overline{3}): L=2 \otimes S_{q_{1} q_{2}}=0 \quad \Longrightarrow J_{l}=2: \Lambda_{c 2}\left(\frac{3}{2}^{+}, \frac{5}{2}^{+}\right) \quad \Xi_{c 2}\left(\frac{3}{2}^{+}, \frac{5}{2}^{+}\right)$

(b) $l_{\rho}=2, l_{\lambda}=0$

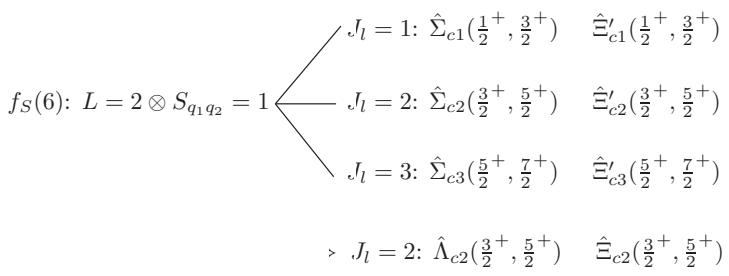

(c) $l_{\rho}=1, l_{\lambda}=1$

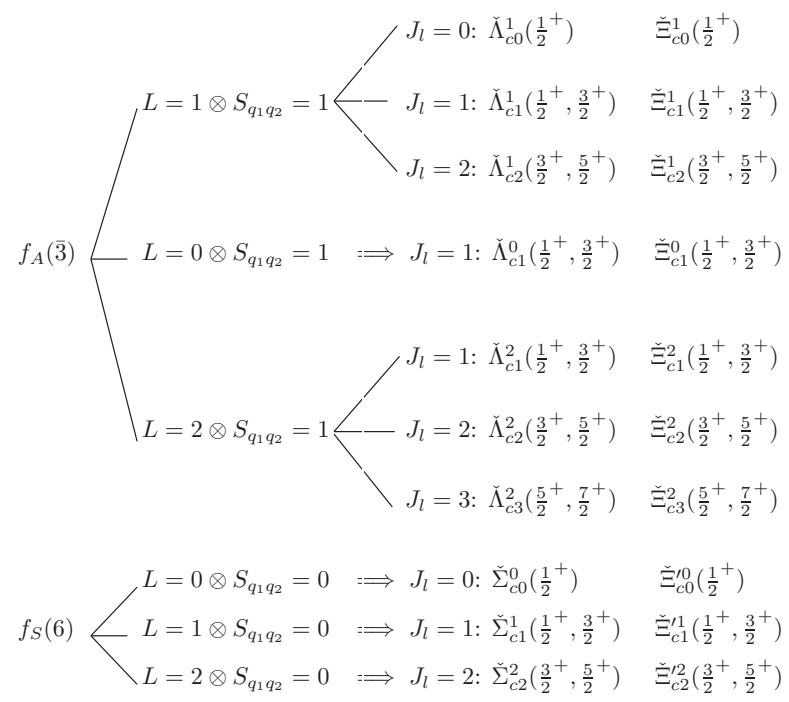

\section{Acknowledgments}

This project was supported by the National Natural Science Foundation of China under Grants 10421503, 10625521, 10705001, and the China Postdoctoral Science foundation (20060400376).

FIG. 1: The notations for the D-wave charmed baryons.

[1] Babar Collaboration, T. Schröder, talk given at the EPS High Energy Physics Conference, Manchester, July, 2007.

[2] BABAR Collaboration, B. Aubert et al., arXiv: hep-ex/0607042.

[3] BELLE Collaboration, R. Chistov et al., Phys. Rev. Lett. 97, 162001 (2006).

[4] BABAR Collaboration, B. Aubert et al., Phys. Rev. Lett. 97, 232001(2006).

[5] BABAR Collaboration, B. Aubert et al., Phys. Rev. Lett. 98, 012001 (2007).

[6] BELLE Collaboration, R. Mizuk et al., Phys. Rev. Lett. 98, 262001 (2007).

[7] C. Chen, X.L. Chen, X. Liu, W.Z. Deng, and S.L. Zhu, Phys. Rev. D 75, 094017 (2007).

[8] L. Micu, Nucl. Phys. B10, 521 (1969).
[9] A. Le Yaouanc, L. Oliver, O. Pène and J. Raynal, Phys. Rev. D8, 2223 (1973); D9, 1415 (1974); D11, 1272 (1975); Phys. lett. B71, 57 (1977); B71, 397 (1977).

[10] A. Le Yaouanc, L. Oliver, O. Pène and J. Raynal, Phys. Lett. B72, 57 (1977).

[11] A. Le Yaouanc, L. Oliver, O. Pène and J. Raynal, Hadron Transitions in the Quark Model, Gordon and Breach Science Publishers, New York, 1987.

[12] H.G. Blundell and S. Godfrey, Phys. Rev. D53, 3700 (1996).

[13] P.R. Page, Nucl. Phys. B446, 189 (1995); S. Capstick and N. Isgur, Phys. Rev. D34, 2809 (1986).

[14] S. Capstick and W. Roberts, Phys. Rev. D49, 4570 (1994).

[15] E.S. Ackleh, T. Barnes and E.S. Swanson, Phys. Rev. 
D54, 6811 (1996).

[16] H.Q. Zhou, R.G. Ping and B.S. Zou, Phys. Lett. B611, $123(2005)$.

[17] X.H. Guo, H.W. Ke, X.Q. Li, X. Liu and S.M. Zhao, Commun. Theor. Phys. 48, 509-518 (2007).

[18] J. Lu, W.Z. Deng, X.L. Chen and S.L. Zhu, Phys. Rev. D 73 054012, (2006); B. Zhang, X. Liu and S.L. Zhu, Eur. Phys. J. C 50, 617 (2007).
[19] S. Capstick and W. Roberts, Phys. Rev. D 47, 1994 (1993).

[20] H. G. Blundell, S. Godfrey, Phys. Rev. D 53, 3700 (1996).

[21] F.E. Close and E.S. Swanson, Phys. Rev. D 72, 094004 (2005).

[22] S. Capstick and N. Isgur, Phys. Rev. D 34, 2809 (1986).

TABLE I: The decay widths of $\Xi_{c}^{+}(3055)$ with different D-wave assignments. Here we list the results with the typical values $\alpha_{\rho}=0.6 \mathrm{GeV}$ and $\alpha_{\lambda}=0.6 \mathrm{GeV}$.

\begin{tabular}{|c|c|c|c|c|c|c|c|c|}
\hline Assignment & $\Xi_{c}^{0} \pi^{+}$ & $\Xi_{c}^{\prime 0} \pi^{+}$ & $\Xi_{c}^{\star 0} \pi^{+}$ & $\Sigma_{c}^{++} k^{-}$ & $\Sigma_{c}^{\star++} k^{-}$ & $\Lambda_{c}^{+} k^{0}$ & $D^{+} \Lambda$ & Remark \\
\hline$\Xi_{c 2}\left(\frac{3}{2}^{+}\right)$ & 0.0 & 1.9 & 0.25 & 2.2 & 0.12 & 0.0 & 0.0 & \\
\hline$\Xi_{c 2}\left(\frac{5}{2}^{+}\right)$ & 0.0 & 0.028 & 1.4 & $0.83 \times 10^{-2}$ & 0.69 & 0.0 & 0.0 & \\
\hline$\Xi_{c 1}^{\prime}\left(\frac{1}{2}^{+}\right)$ & 6.4 & 1.3 & 0.38 & 1.5 & 0.19 & 8.0 & 2.4 & \\
\hline$\Xi_{c 1}^{\prime}\left(\frac{3}{2}^{+}\right)$ & 6.4 & 0.32 & 0.96 & 0.37 & 0.48 & 8.0 & 2.4 & \\
\hline$\Xi_{c 2}^{\prime}\left(\frac{3}{2}^{+}\right)$ & 0.0 & 2.9 & 0.36 & 3.3 & 0.17 & 0.0 & 0.0 & \\
\hline$\Xi_{c 2}^{\prime}\left(\frac{5}{2}^{+}\right)$ & 0.0 & 0.019 & 2.1 & $0.55 \times 10^{-2}$ & 1.0 & 0.0 & 0.0 & \\
\hline$\Xi_{c 3}^{\prime}\left(\frac{5}{2}^{+}\right)$ & 0.15 & 0.022 & $0.78 \times 10^{-2}$ & $0.63 \times 10^{-2}$ & $0.30 \times 10^{-3}$ & 0.18 & 0.0067 & $\times$ \\
\hline$\Xi_{c 3}^{\prime}\left(\frac{7}{2}^{+}\right)$ & 0.15 & 0.012 & 0.011 & $0.35 \times 10^{-2}$ & $0.41 \times 10^{-3}$ & 0.18 & 0.0067 & $x$ \\
\hline$\hat{\Xi}_{c 2}\left(\frac{3}{2}^{+}\right)$ & 0.0 & 27.4 & 21.3 & 14.4 & 2.5 & 0.0 & 0.0 & $x$ \\
\hline$\hat{\Xi}_{c 2}\left(\frac{5}{2}^{+}\right)$ & 0.0 & 27.4 & 21.3 & 14.4 & 2.5 & 0.0 & 0.0 & $\times$ \\
\hline${\hat{\Xi^{\prime}}}_{c 1}\left(\frac{1}{2}^{+}\right)$ & 163 & 18.3 & 3.5 & 9.6 & 0.41 & 205 & 15.5 & $x$ \\
\hline${\hat{\Xi^{\prime}}}_{c 1}\left(\frac{3}{2}^{+}\right)$ & 163 & 4.6 & 8.9 & 2.4 & 1.0 & 205 & 15.5 & $\times$ \\
\hline${\hat{\Xi^{\prime}}}_{c 2}\left(\frac{3}{2}^{+}\right)$ & 0.0 & 41.1 & 15.9 & 21.5 & 1.9 & 0.0 & 0.0 & $x$ \\
\hline$\hat{\Xi}_{c 2}^{\prime}\left(\frac{5}{2}^{+}\right)$ & 0.0 & 18.3 & 24.8 & 9.6 & 2.9 & 0.0 & 0.0 & $\times$ \\
\hline${\hat{\Xi^{\prime}}}_{c 3}\left(\frac{5}{2}^{+}\right)$ & 105 & 20.9 & 10.1 & 10.9 & 1.2 & 131 & 10.0 & $x$ \\
\hline$\hat{\Xi}_{c 3}^{\prime}\left(\frac{7}{2}^{+}\right)$ & 105 & 11.7 & 13.7 & 6.1 & 1.6 & 131 & 10.0 & $\times$ \\
\hline$\check{\Xi}_{c 0}^{\prime}\left(\frac{1}{2}^{+}\right)$ & 0.0 & 0.23 & 0.46 & 1.9 & 2.9 & 0.0 & 0.0 & \\
\hline$\check{\Xi}_{c 1}^{0}\left(\frac{1}{2}^{+}\right)$ & 9.8 & 0.30 & 0.15 & 2.6 & 0.95 & 12.4 & 0.60 & \\
\hline$\check{\Xi}_{c 1}^{0}\left(\frac{3}{2}^{+}\right)$ & 9.8 & 0.075 & 0.38 & 0.65 & 2.4 & 12.4 & 0.60 & \\
\hline$\check{\Xi}_{c 1}^{\prime}\left(\frac{1}{2}^{+}\right)$ & 0.0 & 34.7 & 9.8 & 36.0 & 4.1 & 0.0 & 0.0 & $x$ \\
\hline$\check{\Xi}_{c 1}^{\prime 1}\left(\frac{3}{2}^{+}\right)$ & 0.0 & 8.7 & 24.4 & 9.0 & 10.3 & 0.0 & 0.0 & $x$ \\
\hline$\check{\Xi}_{c 0}^{1}\left(\frac{1}{2}^{+}\right)$ & 0.0 & 34.7 & 39.1 & 36.0 & 16.6 & 0.0 & 0.0 & $x$ \\
\hline$\check{\Xi}_{c 1}^{1}\left(\frac{1}{2}^{+}\right)$ & 97.6 & 17.4 & 4.9 & 18.0 & 2.1 & 122 & 28.2 & $\times$ \\
\hline$\check{\Xi}_{c 1}^{1}\left(\frac{3}{2}^{+}\right)$ & 97.6 & 4.3 & 12.2 & 4.5 & 5.2 & 122 & 28.2 & $x$ \\
\hline$\check{\Xi}_{c 2}^{1}\left(\frac{\overline{3}}{2}^{+}\right)$ & 0.0 & 21.7 & 2.4 & 22.5 & 1.0 & 0.0 & 0.0 & $\times$ \\
\hline$\check{\Xi}_{c 2}^{1}\left(\frac{5}{2}^{+}\right)$ & 0.0 & 0.0 & 14.7 & 0.0 & 6.2 & 0.0 & 0.0 & \\
\hline$\check{\Xi}_{c 2}^{\prime 2}\left(\frac{3}{2}^{+}\right)$ & 0.0 & 8.6 & 4.7 & 12.3 & 1.5 & 0.0 & 0.0 & \\
\hline$\check{\Xi}_{c 2}^{\prime 2}\left(\frac{5}{2}^{+}\right)$ & 0.0 & 4.7 & 8.7 & 2.8 & 4.4 & 0.0 & 0.0 & \\
\hline$\check{\Xi}_{c 1}^{2}\left(\frac{1}{2}^{+}\right)$ & 21.9 & 5.7 & 2.0 & 8.2 & 1.1 & 27.2 & 12.2 & $x$ \\
\hline$\check{\Xi}_{c 1}^{2}\left(\frac{3}{2}^{+}\right)$ & 21.9 & 1.4 & 4.9 & 2.1 & 2.8 & 27.2 & 12.2 & $x$ \\
\hline$\check{\Xi}_{c 2}^{2}\left(\frac{3}{2}^{+}\right)$ & 0.0 & 12.9 & 4.1 & 18.5 & 1.5 & 0.0 & 0.0 & $x$ \\
\hline$\check{\Xi}_{c 2}^{2}\left(\frac{5}{2}^{+}\right)$ & 0.0 & 3.2 & 11.7 & 1.9 & 6.3 & 0.0 & 0.0 & \\
\hline$\check{\Xi}_{c 3}^{2}\left(\frac{\overline{5}}{2}^{+}\right)$ & 17.4 & 3.6 & 1.9 & 2.2 & 0.41 & 21.9 & 2.1 & $\times$ \\
\hline$\check{\Xi}_{c 3}^{2}\left(\frac{7}{2}^{+}\right)$ & 17.4 & 2.0 & 2.5 & 1.2 & 0.57 & 21.9 & 2.1 & $\times$ \\
\hline
\end{tabular}


TABLE II: The decay widths of $\Xi_{c}^{+}(3123)$ with different D-wave assignments. Here we list the results with the typical values $\alpha_{\rho}=0.6 \mathrm{GeV}$ and $\alpha_{\lambda}=0.6 \mathrm{GeV}$.

\begin{tabular}{|c|c|c|c|c|c|c|c|c|}
\hline \begin{tabular}{c|} 
Assignment \\
\end{tabular} & $\Xi_{c}^{0} \pi^{+}$ & $\Xi_{c}^{\prime 0} \pi^{+}$ & $\Xi_{c}^{\star 0} \pi^{+}$ & $\Sigma_{c}^{++} k^{-}$ & $\Sigma_{c}^{\star++} k^{-}$ & $\Lambda_{c}^{+} k^{0}$ & $D^{+} \Lambda$ & Remark \\
\hline$\Xi_{c 2}\left(\frac{3}{2}{ }^{+}\right)$ & 0.0 & 2.8 & 0.43 & 4.5 & 0.49 & 0.0 & 0.0 & \\
\hline$\Xi_{c 2}\left(\frac{5}{2}^{+}\right)$ & 0.0 & 0.075 & 2.3 & 0.053 & 2.8 & 0.0 & 0.0 & \\
\hline$\Xi_{c 1}^{\prime}\left(\frac{1}{2}^{+}\right)$ & 8.3 & 1.9 & 0.63 & 3.0 & 0.79 & 10.2 & 5.5 & $\times$ \\
\hline$\Xi_{c 1}^{\prime}\left(\frac{3}{2}^{+}\right)$ & 8.3 & 0.46 & 1.6 & 0.76 & 2.0 & 10.2 & 5.5 & $\times$ \\
\hline$\Xi_{c 2}^{\prime}\left(\frac{3}{2}^{+}\right)$ & 0.0 & 4.2 & 0.60 & 6.8 & 0.72 & 0.0 & 0.0 & \\
\hline$\Xi_{c 2}^{\prime}\left(\frac{5}{2}^{+}\right)$ & 0.0 & 0.050 & 3.4 & 0.035 & 4.3 & 0.0 & 0.0 & \\
\hline$\Xi_{c 3}^{\prime}\left(\frac{5}{2}^{+}\right)$ & 0.32 & 0.057 & 0.026 & 0.040 & 0.010 & 0.44 & 0.069 & $x$ \\
\hline$\Xi_{c 3}^{\prime}\left(\frac{7}{2}^{+}\right)$ & 0.32 & 0.032 & 0.035 & 0.023 & 0.013 & 0.44 & 0.069 & $\times$ \\
\hline$\hat{\vec{\Xi}}_{c 2}\left(\frac{3}{2}{ }^{+}\right)$ & 0.0 & 58.9 & 53.0 & 56.0 & 30.0 & 0.0 & 0.0 & $\times$ \\
\hline$\hat{\Xi}_{c 2}\left(\frac{5}{2}^{+}\right)$ & 0.0 & 58.9 & 53.0 & 56.0 & 30.0 & 0.0 & 0.0 & $\times$ \\
\hline$\hat{\Xi}_{c 1}^{\prime}\left(\frac{1}{2}^{+}\right)$ & 311 & 39.2 & 8.8 & 37.4 & 5.0 & 411 & 85.9 & $\times$ \\
\hline$\hat{\Xi}_{c 1}^{\prime}\left(\frac{3}{2}^{+}\right)$ & 311 & 9.8 & 22.1 & 9.3 & 12.5 & 411 & 85.9 & $\times$ \\
\hline${\hat{\Xi^{\prime}}}_{c 2}\left(\frac{\overline{3}}{2}^{+}\right)$ & 0.0 & 88.3 & 40.0 & 84.1 & 22.5 & 0.0 & 0.0 & $\times$ \\
\hline$\hat{\Xi}_{c 2}^{\prime}\left(\frac{5}{2}^{+}\right)$ & 0.0 & 39.2 & 61.8 & 37.4 & 35.0 & 0.0 & 0.0 & $\times$ \\
\hline$\hat{\Xi}_{c 3}^{\prime}\left(\frac{5}{2}^{+}\right)$ & 200 & 44.8 & 25.2 & 42.7 & 14.3 & 264 & 55.3 & $\times$ \\
\hline$\hat{\Xi}_{c 3}^{\prime}\left(\frac{7}{2}^{+}\right)$ & 200 & 25.2 & 34.0 & 24.0 & 19.3 & 264 & 55.3 & $\times$ \\
\hline$\check{\Xi}_{c 0}^{\prime}\left(\frac{1}{2}^{+}\right)$ & 0.0 & 4.3 & 0.35 & 0.015 & 2.5 & 0.0 & 0.0 & \\
\hline$\check{\Xi}_{c 1}^{0}\left(\frac{1}{2}^{+}\right)$ & 36.5 & 5.8 & 0.12 & 0.020 & 0.82 & 52.7 & 1.8 & $x$ \\
\hline$\check{\Xi}_{c 1}^{0}\left(\frac{3}{2}^{+}\right)$ & 36.5 & 1.4 & 0.29 & 0.005 & 2.0 & 52.7 & 1.8 & $\times$ \\
\hline$\check{\Xi}_{c 1}^{\prime}\left(\frac{1}{2}^{+}\right)$ & 0.0 & 54.3 & 17.0 & 80.2 & 18.3 & 0.0 & 0.0 & $\times$ \\
\hline$\check{\Xi}_{c 1}^{\prime 1}\left(\frac{3}{2}^{+}\right)$ & 0.0 & 13.6 & 42.6 & 20.1 & 45.8 & 0.0 & 0.0 & $\times$ \\
\hline$\check{\Xi}_{c 0}^{1}\left(\frac{1}{2}^{+}\right)$ & 0.0 & 54.3 & 68.2 & 80.2 & 73.3 & 0.0 & 0.0 & $\times$ \\
\hline$\check{\Xi}_{c 1}^{1}\left(\frac{1}{2}^{+}\right)$ & 139 & 27.1 & 8.5 & 40.1 & 9.2 & 175 & 73.3 & $\times$ \\
\hline$\check{\Xi}_{c 1}^{1}\left(\frac{3}{2}^{+}\right)$ & 139 & 6.8 & 21.3 & 10.0 & 22.9 & 175 & 73.3 & $\times$ \\
\hline$\check{\Xi}_{c 2}^{1}\left(\frac{3}{2}^{+}\right)$ & 0.0 & 33.9 & 4.3 & 50.1 & 4.6 & 0.0 & 0.0 & $\times$ \\
\hline$\check{\Xi}_{c 2}^{1}\left(\frac{5}{2}^{+}\right)$ & 0.0 & 0.0 & 25.6 & 0.0 & 27.5 & 0.0 & 0.0 & $\times$ \\
\hline$\check{\Xi}_{c 2}^{\prime 2}\left(\frac{3}{2}^{+}\right)$ & 0.0 & 10.5 & 10.0 & 21.3 & 8.1 & 0.0 & 0.0 & $\times$ \\
\hline$\check{\Xi}_{c 2}^{\prime 2}\left(\frac{5}{2}^{+}\right)$ & 0.0 & 9.9 & 13.9 & 9.8 & 16.9 & 0.0 & 0.0 & $\times$ \\
\hline$\check{\Xi}_{c 1}^{2}\left(\frac{1}{2}^{+}\right)$ & 21.6 & 7.0 & 2.8 & 14.2 & 4.0 & 25.2 & 21.1 & $\times$ \\
\hline$\check{\Xi}_{c 1}^{2}\left(\frac{3}{2}^{+}\right)$ & 21.6 & 1.7 & 7.1 & 3.6 & 10.0 & 25.2 & 21.1 & $\times$ \\
\hline$\check{\Xi}_{c 2}^{2}\left(\frac{3}{2}^{+}\right)$ & 0.0 & 15.7 & 8.1 & 32.0 & 7.4 & 0.0 & 0.0 & $\times$ \\
\hline$\check{\Xi}_{c 2}^{2}\left(\frac{5}{2}^{+}\right)$ & 0.0 & 6.6 & 17.7 & 6.6 & 23.2 & 0.0 & 0.0 & $\times$ \\
\hline$\check{\Xi}_{c 3}^{2}\left(\frac{\overline{5}}{2}^{+}\right)$ & 32.7 & 7.5 & 4.4 & 7.5 & 3.0 & 43.1 & 10.1 & $\times$ \\
\hline$\check{\Xi}_{c 3}^{2}\left(\frac{7}{2}^{+}\right)$ & 32.7 & 4.2 & 5.9 & 4.2 & 4.1 & 43.1 & 10.1 & $\times$ \\
\hline
\end{tabular}

